\title{
Definitions Management: A Semantics-Based Approach for Clinical Documentation in Healthcare Delivery
}

\author{
Vipul Kashyap ${ }^{1}$, Alfredo Morales ${ }^{2}$, Tonya Hongsermeier ${ }^{1}$, and Qi Li ${ }^{1}$ \\ ${ }^{1}$ Clinical Informatics R\&D, Partners Healthcare System, 93 Worcester St, \\ Suite 201, Wellesley, MA 02481, USA \\ \{vkashyap1, thongsermeier, qli5\} apartners.org \\ ${ }^{2}$ Cerebra, 5963 La Place Court, Suite 200, Carlsbad, CA 92008, USA \\ Alfredo.Morales@cerebra.com
}

\begin{abstract}
Structured Clinical Documentation is a fundamental component of the healthcare enterprise, linking both clinical (e.g., electronic health record, clinical decision support) and administrative functions (e.g., evaluation and management coding, billing). Documentation templates have proven to be an effective mechanism for implementing structured clinical documentation. The ability to create and manage definitions, i.e., definitions management, for various concepts such as diseases, drugs, contraindications, complications, etc. is crucial for creating and maintaining documentation templates in a consistent and cohesive manner across the organization. Definitions management involves the creation and management of concepts that may be a part of controlled vocabularies, domain models and ontologies. In this paper, we present a realworld implementation of a semantics-based approach to automate structured clinical documentation based on a description logics (DL) system for ontology management. In this context we will introduce the ontological underpinnings on which clinical documents are based, namely the domain, document and presentation ontologies. We will present techniques that leverage these ontologies to render static and dynamic templates that contain branching logic. We will also evaluate the role of these ontologies in the context of managing the impact of definition changes on the creation and rendering of these documentation templates, and the ability to retrieve documentation templates and their instances precisely in a given clinical context.
\end{abstract}

\section{Introduction}

Structured Clinical Documentation is a fundamental component of the healthcare enterprise linking both clinical (e.g., electronic health record, clinical decision support) and administrative functions (e.g., evaluation and management coding, billing). At its core, Structured Clinical Documentation consists of template-based, logically driven instruments designed to facilitate and minimize the guesswork of coding and documenting information regarding a patient throughout the continuum of care. When well implemented in the context of the clinical workflow, these instruments can save clinicians' time as well as well as assure clinical thoroughness, thus reducing the chance of medical errors.

Stakeholders in basic sciences, health services and medical informatics research recognize the importance of information captured directly from episodes of clinical 
care for research, real-time decision support and patient screening for clinical trials or clinical syndromes $[1,2]$. Structured entry and reporting systems promise to meet this need by enabling health care providers to document clinical encounters through selection from pre-defined categories [3-6].

Structured entry and reporting systems are designed to enhance the process of clinical documentation by both presenting useful categorical concepts in a user interface and capturing input from the end user though the interface as machine-readable data. Structured entry is achieved as the end user navigates through lists of relevant concepts and sets status (e.g. present/absent, etc.) or strongly typed values. The main objectives of these systems are:

- Capture of raw data in a consistent and cohesive manner enabling generation and delivery of reminders and alerts to the point of care while providing an infrastructure for clinical research. This leads to enhancement of patient care.

- Reduce variability in the quality and quantity of concepts recorded as part of the medical record. This enables research investigators to seamlessly integrate research questions without impacting the clinical workflow.

Although the benefits of structured entry and reporting systems have been well documented, their widespread implementation and consequent adoption has been limited due to the following reasons [8-11]:

- Inefficiency, complexity and slow pace of navigating through user interfaces to find relevant content

- Inflexibility for documenting unforeseen findings

- Lack of integration with clinical applications

- Deficiencies in both coverage by and goals of the underlying domain and document models.

- Lack of consistency and maintenance of documentation templates in the context of evolving domain and document models.

- Decreased overall efficiency for generating complex documents.

We present a semantics-based infrastructure that seeks to address some of the abovementioned issues. The ability to create and manage definitions, i.e., definitions management, for various concepts such as diseases, drugs, complications, etc. is crucial for consistent maintenance and creation of documentation templates across the organization. Definitions management involves the creation and management of concepts and other knowledge objects that could be components of controlled vocabularies, domain models and ontologies. In this paper, we present a real world implementation of a semantics-based approach, with ontological underpinnings to automate execution and maintenance of documentation templates based on description logics (DL) system for ontology management.

The ontological underpinnings of the proposed infrastructure help address issues related to the evolution of the underlying document and domain models. We leverage ontologies and semantic inferences to automate the process of generating and rendering complex documents and enable precise location of relevant clinical content referenced in these documents. These techniques enable rendering static and dynamic templates that contain branching logic and manage the impact of definition changes on the creation and rendering of these documentation templates. 
The organization of the paper is as follows. In Section 2, we present a real-world scenario and use case that describes an actual documentation template implemented at Partners HealthCare. Section 3 discusses the document and domain ontologies used in the system. We present and discuss the architecture of the system in Section 4. In Sections 5 and 6 , we present the functionality of the clinical documentation system in the clinical and maintenance contexts. The role of semantic inference is explored in Section 7. Section 8 presents conclusions and future work.

\section{Use Case and Scenario}

A sample clinical documentation template being implemented at Partners HealthCare System is presented. It consists of a set of questions that elicit information related to patient state, including diseases, active medications and other important clinical information such as lab results and conditions or situations that makes a given therapy, treatment or procedure inadvisable (this is known as contraindications.)

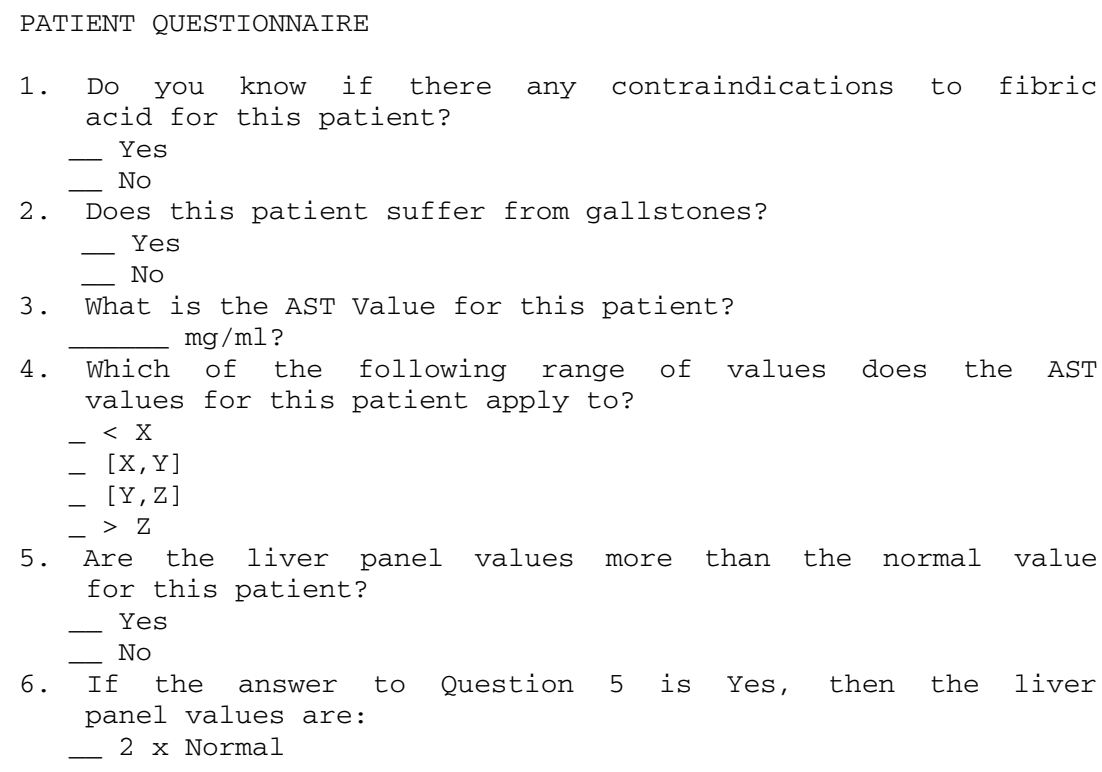

The above instrument contains a set of data collection items, each of which attempts to elicit some information about the patient. It may be noted that some of the information might either be elicited directly from the physician and some of it may be populated from the Electronic Medical Record (EMR).

The conventional approach to automate this template involves the design of tightly coupled structures incorporating information to be collected embedded within the business logic and presentation logic required to present them to the end user. Variations of this approach attempt to separate presentation logic from business logic and the data structures where the captured information is stored. 
Adopting either approach poses significant challenges. The maintainability and extensibility of the resulting documentation template may require significant additional investment as changes in the information requirements occur along with changes in presentation and business logic requirements. A different approach, based on the composition of the underlying information and domain knowledge models based on their underlying semantics, is needed to facilitate the development and delivery of documentation templates as adaptive knowledge assets. If we adopt the above approach to defining and creating a template, the following observations can be made:

- A distinction needs to be made between the questions being asked and the information about which the enquiry is being made. For instance, in Question 1, there is a distinction between contraindication to fibric acid as opposed to does this patient have contraindication to fibric acid?

- The values displayed as potential answers to a question may not cover all the possible values associated with an information item. For instance, in Question 4, the values displayed as potential responses for AST may not cover all potential values of $A S T$ observed in practice. This template may be intended for a specialized subset of patients, which could be reflected in the potential choices available.

- Most questions on the template are related to some attributes of the patient state. For instance, various questions in the questionnaire refer to contraindications, clinical conditions (suffer), clinical laboratory tests (AST, Liver Panel)

- There is branching logic embedded in this questionnaire. For instance, Question 6 becomes relevant only when the answer to Question 5 is Yes. This is just a simple example. In general there might be complicated patterns for branching logic that might be represented as a graph or a tree structure.

- Some of the information being discussed in the question might be composite in structure. For instance, the information item, Liver Panel in Question 5 consists of a set of laboratory tests that are used to determine the liver condition of a patient

- Some of the values displayed as potential answers to the questions may be $d e$ rived from the actual values. For instance in Question 6, the answers are based on the normal AST (e.g., $2 \times$ Normal), where normal describes the range of values for that given laboratory test that are considered within the norm. These values may either be derived via statistical processes or stored as default values associated with the information item. The latter is typically the case in biomedical information systems such as an EMR

- Some of the answers to a question might be intervals of values. This is illustrated in Question 4

The observations above suggest a need to delineate between information related to the document template (questions, attributes, values) and information related to the biomedical concepts referred to in the documentation template. This helps us identify the two types of ontologies, viz. document and domain ontologies that underpin the system. Functionality needed to enable presentation and rendering of templates can now be specified in terms of operations on the underlying ontologies. In the next section, we illustrate these two types of ontologies represented using OWL-DL expressions that were constructed using the Cerebra Construct workbench. 


\section{Ontological Underpinnings}

The content and structure of a documentation template can be decomposed and modeled using three types of ontologies:

Document Ontologies: Documentation aspects of the template, such as the data collection item, the questions being asked, the properties about the patient state about which the question is being asked, the set of proposed responses are modeled using document ontologies. These ontologies conform to a document template meta-model that determines the composition and the logical organization of a document template, and may be viewed as a container framework where biomedical knowledge is referred to and accessed while presenting the template to a physician in the context of a clinical encounter. Fig. 1. illustrates the representation of the documentation template presented in the previous section as a document ontology. This representation was made using Cerebra Construct, a MS Vision add-on that allows modeling of ontologies following a visual paradigm producing OWL-DL as output. In the example, the document template ontology consists of a concept Document corresponding to the template. The Document concept contains concepts such as "Section" which contains concepts related to each of the questions such as Known Contraindications to Fibric Acid?, Known Gallstones History?, etc. Each of these questions contains references to biomedical concepts such as FibricAcidContraindication, Patient, etc.

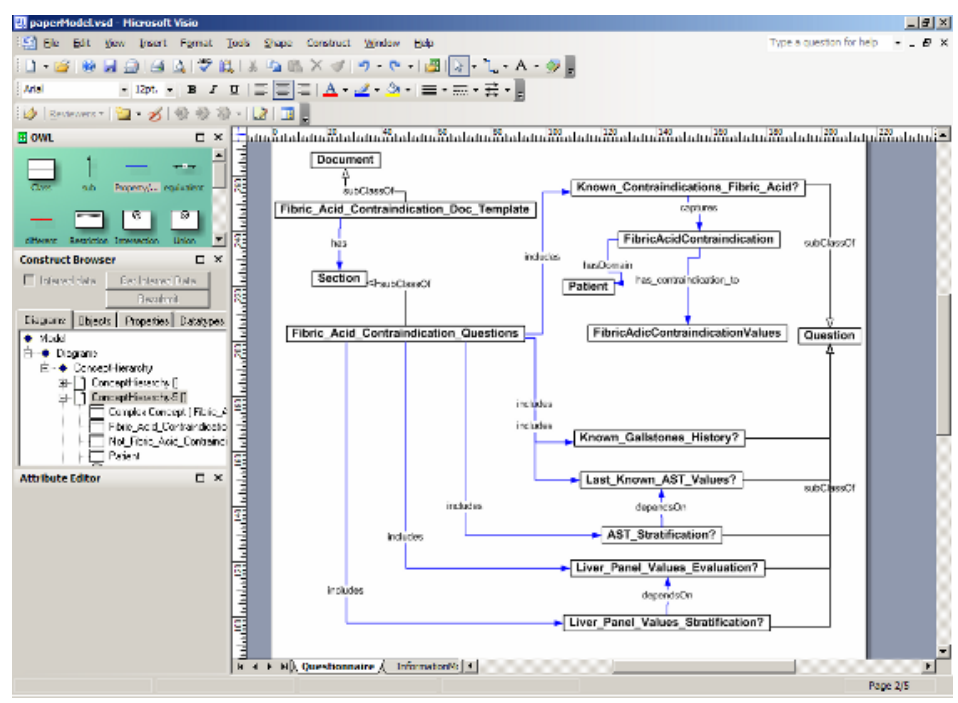

Fig. 1. Sample Documentation Template Ontology as Represented in Cerebra Construct

Domain Ontologies: Biomedical knowledge referenced in the documentation template is modeled using domain specific ontologies. This description will include concepts that may have their origin in controlled vocabularies, e.g., SNOMED CT [16], LOINC [17], ICD-9-CM[18], complex concepts that may arise from their combina- 
tion following a logical prescription, relationships among those concepts as well as restrictions over the concepts imposed by characterizations of their attributes and the values that they may assume. The concepts, restrictions and attributes modeled in the use case are represented in Fig. 2.

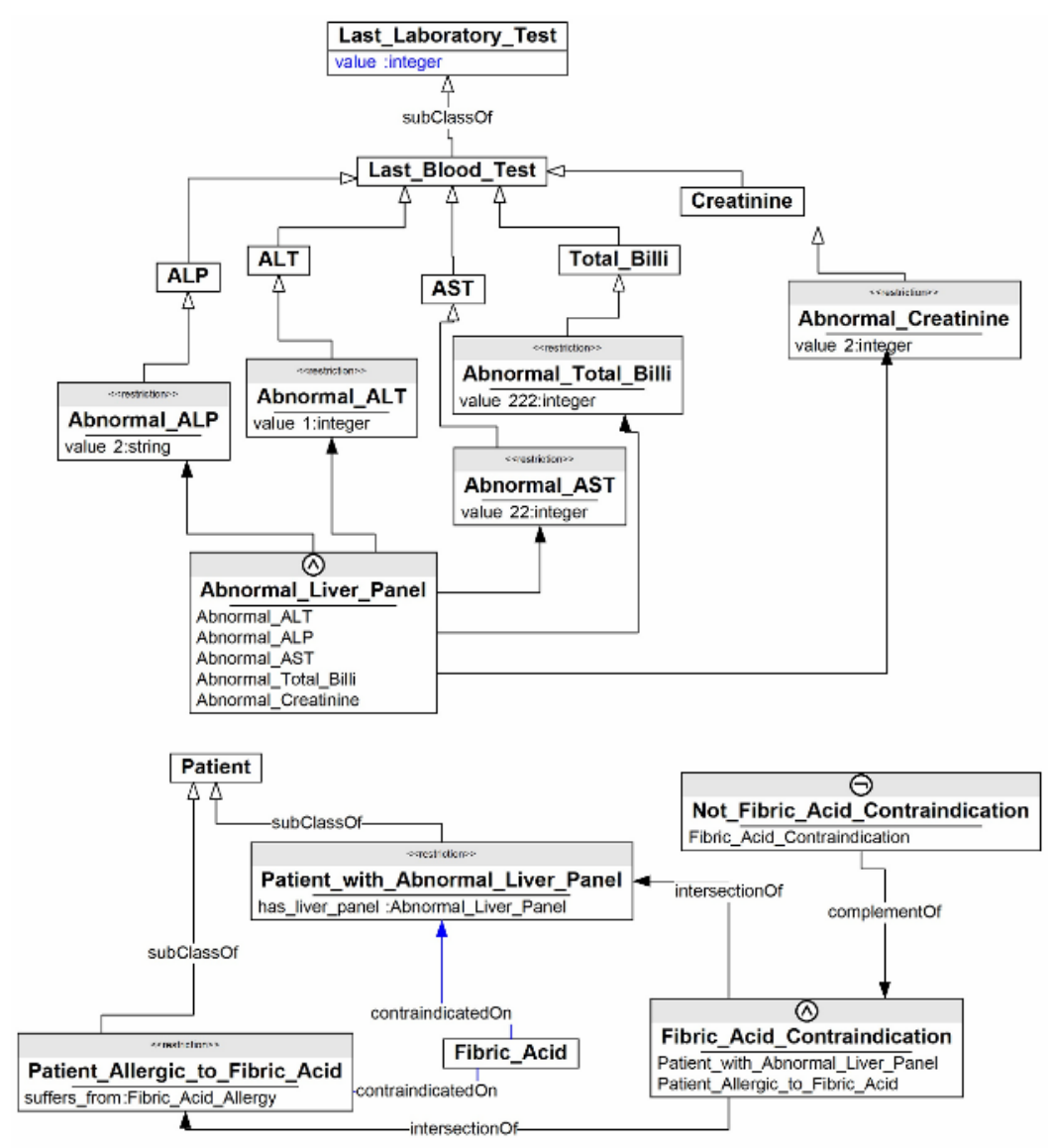

Fig. 2. Representation of Domain Ontology for Sample Documentation Template

- Patient: This refers to the subject of the documentation template which is a patient. The questions in the questionnaire refer to the properties of patient state, such as has_contraindication, which specifies a patient's contraindications to drugs and therapies; suffers_from, which specifies a patient's conditions or problems; has_AST_value, a data type property that specifies the results of an AST test on the patient; has_liver_panel, which specifies liver panel values of a patient.

- Contraindications: This refers to a set of contraindications

- Fibric_Acid: This refers to the compound Fibric Acid 


\section{Information Ontology}

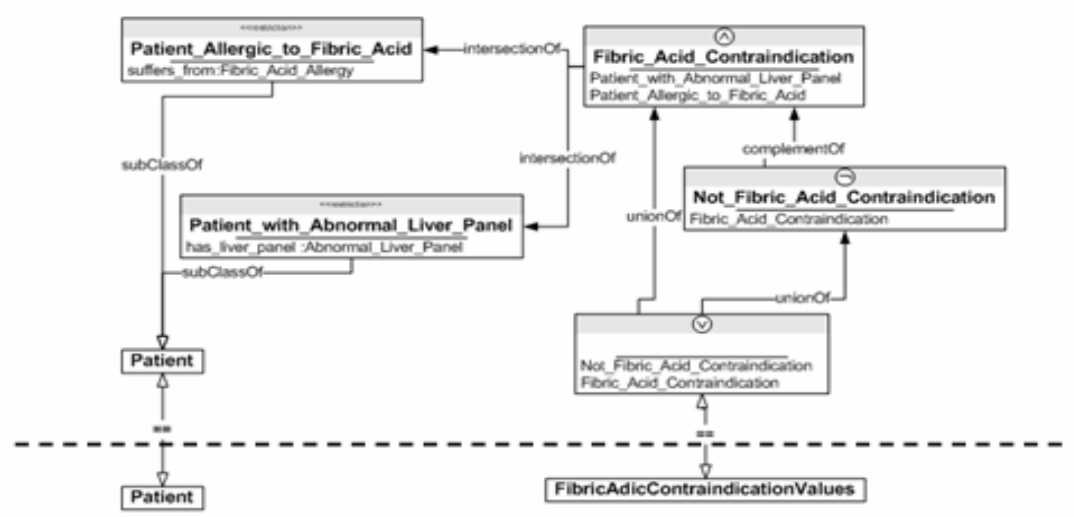

\section{Document Ontology}

Fig. 3. Composition Ontology

- Fibric_Acid_Contraindication: This refers to a subclass of contraindications that deal with Contraindication to Fibric Acid and can be defined as:

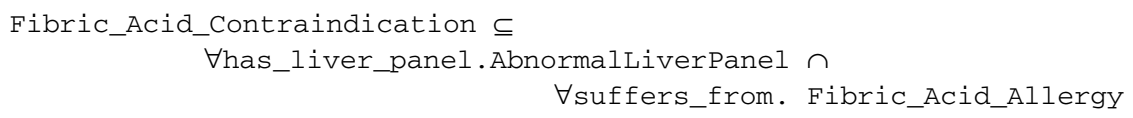

- Gallstones: This refers to the concept Gallstones

- AST_Value: This is a datatype which refers to a range of valid AST values. Cerebra Server provides a mechanism to define ranges as restrictions based on values of the datatype.

- Liver_Panel: This is a composite concept that is a cross product (intersection) of the concepts, ASTValue, ALTValue, AlkalinePhosphateValue, TotalBilirubinValue. The DL expression for LiverPanel can be given as:

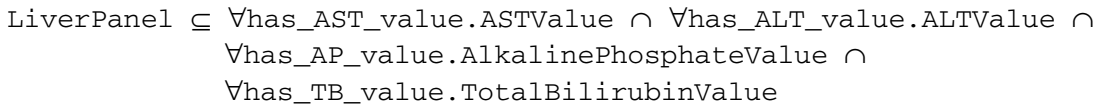

Composition Ontologies: Constitute a set of inter-ontology articulations between elements in the document ontology and domain ontologies. These articulations capture the association between a documentation template and the domain knowledge it contains. Fig. 3. presents the inter-ontology articulations between the document ontology and the domain ontology for the sample documentation template.

\section{Architecture}

The architectural components of the clinical documentation engine being implemented at Partners HealthCare are presented in Fig. 4. 


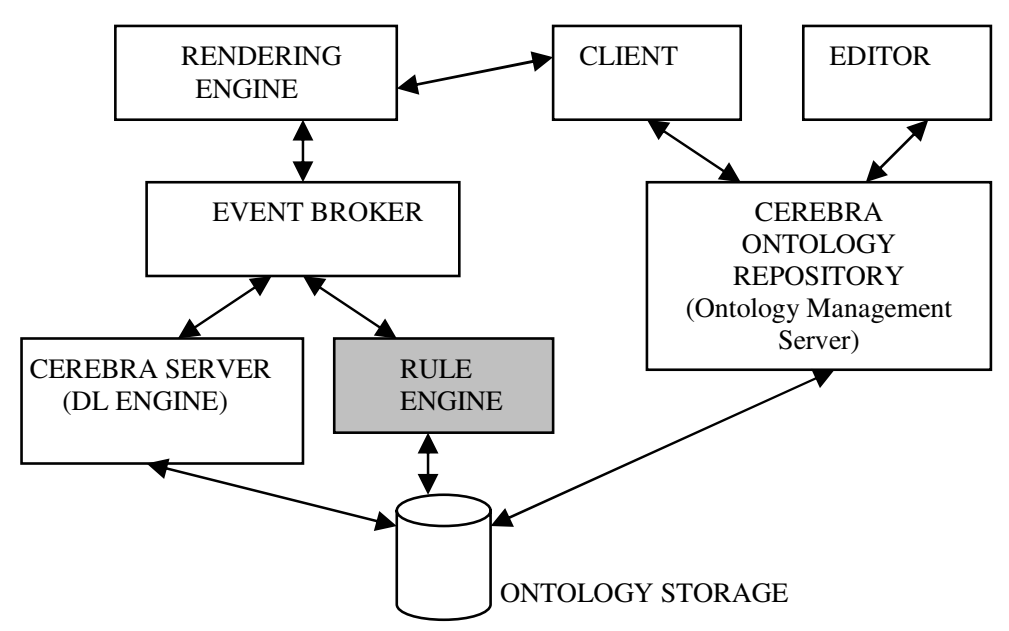

Fig. 4. Clinical Documentation System Architecture

- Client/Editor: User interfaces are needed in tow contexts. In the transactional or execution context, the clinician will enter data into the documentation template. In the maintenance context, the knowledge engineer will make editorial changes to the underlying ontologies and templates.

- Rendering Engine: The rendering engine acts as a presentation logic layer. It consumes the data collection items presented to it by the event broker component, applies style sheet templates and presents them to the user interface.

- Event Broker: The event broker implements a blackboard architecture where it receives a stream of data collection items and decides based on appropriate criteria whether the data collection item needs to be processed by the DL Engine or the Rules Engine. When the event broker receives a patient id it is forwarded to appropriately components to retrieve an initial set of data collection items.

- DL Engine: This component processes OWL-DL based ontologies and provides inferencing capabilities in the execution and maintenance contexts. The DL engine computes potential responses for questions associated with data collection items and identifies redundant questions in the context of interactions between the clinician and the documentation system. In the maintenance context, the DL engine interacts with the Ontology Management Server when the domain and/or document ontologies change, and checks for contradictions and equivalences. The DL engine is implemented by the Cerebra Server.

- Rules Engine: DL engines cannot reason with spatio-temporal information required by some documentation templates. In these cases, the event broker invokes the rules engine to perform the appropriate computations. In the maintenance context, DL engines and Rule engines interact with each other to address changes in ontology and the resulting changes in rule bases (and vice versa). 
- Ontology Management Server: The ontology management server manages changes in the domain and document ontologies in the maintenance context. It propagates the impact of changes in the domain ontology to the document ontology. It invokes the DL engine to check whether the changes introduced are consistent, and to identify new contradictions and equivalences that might surface.

- Ontology Storage: This is the repository which stores the ontologies and instances and might be implemented in a relational database management system.

\section{The Execution Context: Rendering and Instantiation of Documentation Templates}

Documentation templates become alive or are "played" in the execution context, i.e. when a clinician enters data in a documentation template in the context of a clinical encounter with a patient. We assume that the clinician enters the patient id and the documentation system retrieves the appropriate template(s). The DL engine may be invoked to determine the relevant template(s) to be displayed to the clinician. Each new data collection item in the documentation template is viewed as an event, which triggers various computational processes.

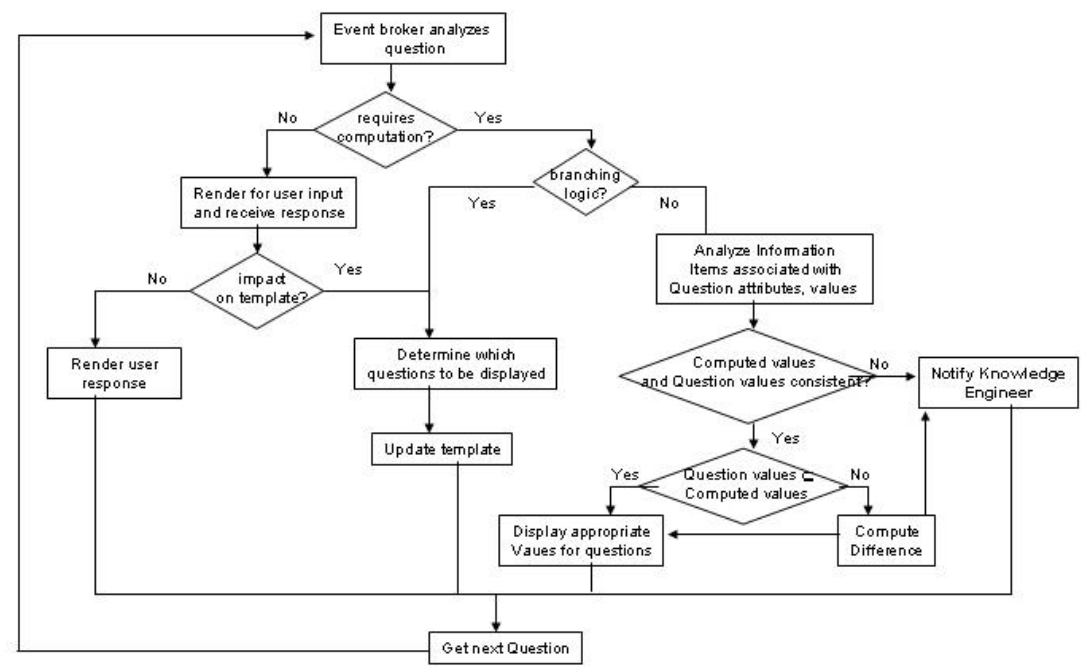

Fig. 5. The Event processing framework

The event processing framework is illustrated in Fig. 5. The workflow provides a mechanism to define how a documentation template will be presented to a clinician at the point of care. It will also evaluate at execution time whether inconsistencies have arisen. These would be communicated to the knowledge engineer, who would address them in maintenance mode. 


\section{The Maintenance Context: Tracking and Maintenance of Domain and Document Ontologies}

In this section, we discuss scenarios related to changes in the definition of domain ontology concept by a knowledge engineer. The ontology management server enables propagation of changes in the domain ontology elements to the relevant document ontology elements as illustrated in the taxonomy in Fig. 6. The possible scenarios in which a knowledge engineer may be notified are:

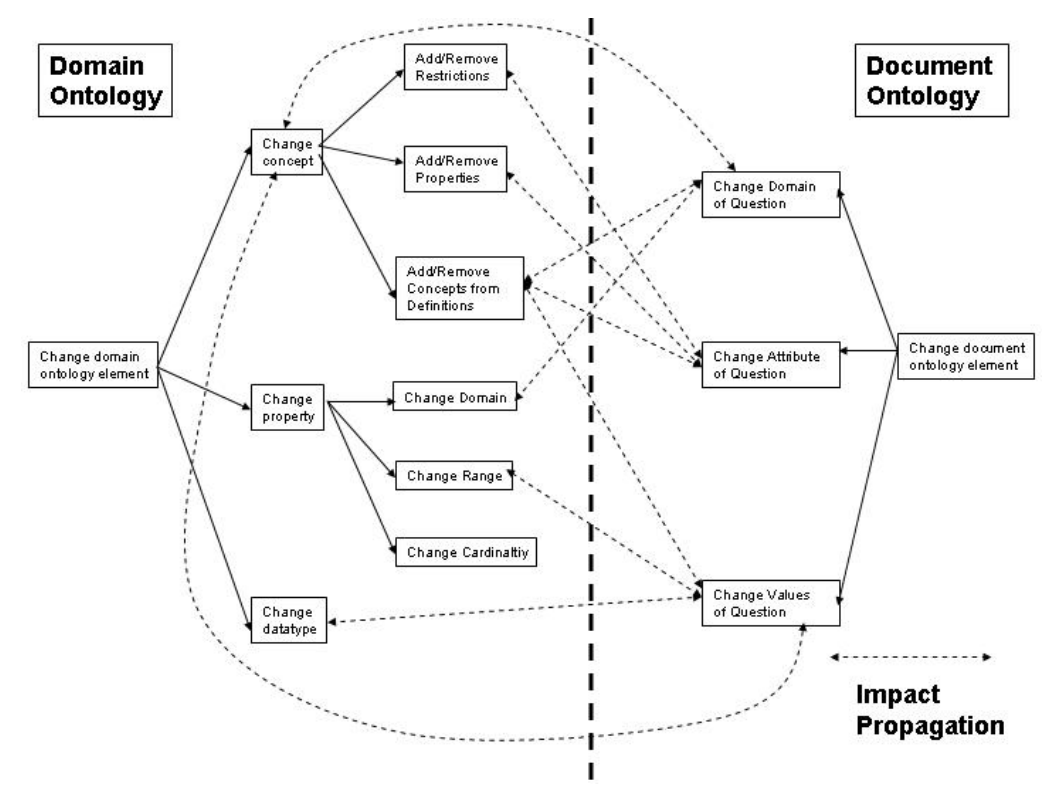

Fig. 6. Taxonomy of Changes in Domain and Document Ontologies

Change in concept definitions: A concept could appear as the domain or range of an attribute (associated with a question) in the document ontology. The knowledge engineer is notified about the relevant attributes and values of a data collection item that are potentially impacted. Potential changes in the domain ontology that can result in changing concept definitions are: adding/removing a restriction on a property, adding/removing of properties and addition of concepts to a definition. For example, one may redefine the concept Patient as SeverelyIllpatient by adding a property severity-level with the restriction that it has a value "high".

$$
\text { Patient changes to Patient } \cap \forall \text { severity-level. }\{\text { "high" \} }
$$

Consider another example, where one may redefine the concept contraindicationToFibricAcid as a disjunction of AbnormalAST and AbnormalALT. i.e., 
Change in property definitions: A property typically appears as an attribute of a data collection item on which a question is posed. The knowledge engineer is notified of the questions impacted by this change. A definition can be changed by changing either its domain, range or cardinality. In each case the knowledge engineer should check that the domain and range associated with the relevant data collection items are consistent with the change. For example if the definition of the concept Patient is changed to FemalePatient, i.e., Patient changes to Patient $\cap$ Female, all the properties of FemalePatient, that appear in a data collection item are flagged and the knowledge engineer is notified. If the cardinality of a property changes from $\leq 1$ to $\geq$ 1 , then the user interface for a data collection item may need to be changed from a single select to multi-select.

Change in datatype definitions: Datatype definitions are represented using XML schema types and can be changed by changing the range of values associated with it. For example the value of normal LDL may be changed from $<100$ to $<70$. The data collection items where these datatypes appear as values are flagged and the knowledge engineer is notified.

\section{The Role of Semantic Inferences}

We now illustrate with examples, the role of semantic inferences in the execution and maintenance contexts.

Semantic Inferences in the Execution Context: The ability to retrieve relevant documentation templates based on properties of the patient state. Templates for patients suffering from Diabetes can be retrieved by performing the following inference:

TBox $=$ Patient $\cap \exists$ suffers-from.Diabetes $\equiv \mathbf{D}$ ?

where $\mathbf{D}$ is the information domain associated with a documentation template.

The ability to determine redundant questions based on a user response. For instance, if a patient has a contraindication to fibric acid, the question that seeks information on whether a patient suffers from gallstones becomes redundant. This redundancy can be identified by performing the following inference.

TBox $\vDash \forall$ suffers-from.Gallstones $\subseteq$

$\forall$ has-contraindication.FibricAcidContraindication ?

The ability to determine whether a question needs to be displayed based on the responses to earlier questions. Consider a patient $\mathrm{P}_{1}$ for which some state properties are known. For question 6 (from the instrument) to be rendered, the answer to question 5 should be yes, i.e., the following statement should be satisfiable:

ABox $\vDash \mathrm{P}_{1} \in$ Patient $\cap \forall$ has-liver-panel.AbnormalliverPanel ?

Semantic Inferences in the Maintenance Context: Whenever concept definitions in the domain ontology change, semantic inferences can be performed to enable:

Checking for consistency and contradictions: The DL engine checks whether the changes are consistent or whether they give rise to contradictions. For instance, clinical evidence may suggest that abnormal AST values are not possible in the presence 
of abnormal ATL values. This may result in the following constraint being added to the TBox with the following inference:

ASTValue $\cap$ ATLValue $\equiv \phi$, TBox / AbnormalLiverPanel $\equiv \phi$

This will have an impact on question 5 in the questionnaire as this will never have a valid response. The Knowledge Engineer will be notified of this possibility

Checking for equivalences: In this scenario, the DL engine checks whether the changes introduce new equivalences. For example, consider the following definition of FibricAcidContraindication:

FibricAcidContraindication $\equiv$

ᄏhas_liver_panel.AbnormalLiverPanel $\cap$ Jsuffers_from.Fibric_Acid_Allergy

Suppose the definition of FibricAcidContraindication changes to:

FibricAcidContraindication' $\equiv$ Ghas_liver_panel.AbnormalLiverPanel

This creates a new equivalence which can be used by the DL engine to infer theequivalence of questions 1 and 5, thus making one of them redundant, as follows:

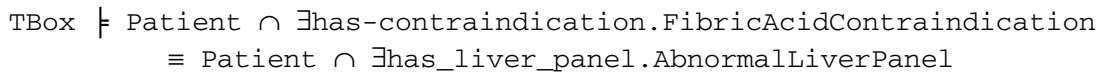

Identifying concept differences: Whenever a concept definition changes, the DL engine can identify the conceptual difference between the two versions of the concept and present the changes to the knowledge engineer. In the example given above, the knowledge engineer will be notified of the following conceptual difference:

TBox $=$ FibricAcidContraindication' $\cap$ Jsuffers_from.Fibric_Acid_Allergy
$\equiv$ FibricAcidContraindication

Datatype reasoning: Whenever the definition of a data type, e.g., AbnormalASTvalues changes, the DL engine can identify potential impacts, including contradictions this can introduce. For instance changing of normal ASTValues from [5,25] to [10, 30] would change the definition of normal LiverPanelvalues which will affect the responses to Questions 5 and 6. These changes will be propagated and the Knowledge Engineer notified.

\section{Conclusions and Future Work}

Clinical documentation templates are an intrinsic part of the process of care in modern medicine and constitute one of the most valuable tools for providing both episodic and preventive care. Mainstream implementations of an electronic documentation template system normally involve representing and storing these ontological concepts and their relationships as fix structures in relational databases or XML stores, exposing them through a middleware layer of enterprise objects (.Net components or EJBs) which functionality would be presented to the end user via a framework of dynamically generated user interfaces.

The approach afore mentioned is intractable over time, as it will require continuous refactoring of enterprise objects and support for specialized data structures to handle 
dynamic knowledge evolution. This will translate to a high cost of ownership and jeopardize their long term viability. In order to cope with the rapid change of knowledge in medicine and its effect on biomedical concepts as illustrated in Sections 6 and 7, technology and techniques are needed that enable model-based dependency propagation and semantic inferences which are not currently supported by various middleware platforms and relational databases.

We have presented a model-based composition method for representing and delivering documentation templates to the point of care. This provides the foundation to define an infrastructure for creation and maintenance of documentation templates, clinical rules and other knowledge assets. Separation of the document model and the domain knowledge is the key architectural design, the adoption of which is expected to enable operational efficiencies at Partners HealthCare System. It is also anticipated that the cost of maintaining clinical documentation templates will be reduced over time generating more return on investment. This is expected to have a positive impact on the ability of the clinician to identify and complete patient relevant documentation in a streamlined manner.

We also introduce a reference semantics-based architecture with ontological underpinnings. The associated ontology management and inferencing capabilities are mechanisms that will enable Partners Healthcare to deliver adaptable documentation templates. These templates will be instantiated based on patient-state based classification. Semantics also provides a mechanism to manage the implications of knowledge change over time, including identification of inconsistencies, equivalences and redundancies that may arise as domain and document ontologies evolve.

It is expected that the modeling approach and implemented architecture, will enable adoption of a scalable, long term solution that integrates seamlessly with the overall knowledge management efforts in progress. This will enable enhancement of documentation templates beyond information gathering nature into a knowledge exploration and delivery mechanism.

A critical requirement for semantics and model based approaches is the availability of semantically rich knowledge that is loaded into the DL engine. Requirements related to clinical documentation will be analyzed and the set of biomedical terminologies and information models that will be identified. The following have been earmarked [14] as a starting set of standards that would constitute the foundational vocabularies and models. These will be represented in the OWL-DL format and preloaded into the DL engine for their use as descriptions of domain knowledge concepts. These standards are:

1. Health Level 7 (HL7) reference framework [15] for structuring and representing clinical information such as demographic information, clinical encounters, observations, diagnosis

2. The College of American Pathologists Systematized Nomenclature of Medicine Clinical Terms (SNOMED CT) [16] for anatomical descriptions, diagnosis documentation, descriptions of interventions and procedures.

3. Laboratory Logical Observation Identifier Name Codes (LOINC) [17] to standardize the electronic exchange of laboratory test orders and drug label section headers.

4. A set of federal terminologies related to medications, including the Food and Drug Administration's names and codes for ingredients, manufactured dosage 
forms, drug products and medication packages, the National Library of Medicine's RxNORM [19] for describing clinical drugs, and the Veterans Administration's National Drug File Reference Terminology (NDF-RT) for specific drug classifications.

5. The Human Gene Nomenclature (HUGN) [20] for exchanging information regarding the role of genes in biomedical research in the federal health sector.

6. The Environmental Protection Agency's Substance Registry System [21] for nonmedicinal chemicals of importance to health care.

The work described in the paper is an ongoing implementation of a definitions management infrastructure for clinical decision support at Partners HealthCare System. Some future initiatives we are looking at are:

- The use of rule-based approaches to capture knowledge not expressible within current DL-based systems

- The integration of the ontology management server with a content management server for a managing the lifecycle of models and ontologies.

- The integration of the rules engine with a content management server for managing the lifecycles of rules in conjunction with their associated models and ontologies.

- Explore the use of description logics that support inferences on spatial and temporal relationships.

\section{References}

1. Committee on Quality of Health Care in America: Using Information Technology. Crossing the Quality Chasm: A New Health System for the $21^{\text {st }}$ Century. Washington, D.C.: IOM; 2001

2. Committee on Improving the Patient Record. The Computer-Based Patient Record: An Essential Technology for Health Care, 2 ed. Washington, DC: ION; 1991

3. Yoder JW, Schultz DF, Williams BT. The MEDIGATE graphical user interface for entry of physical findings: Design principles and implementation. Medical Examination Direct Iconic and Graphic Augmented Text Entry System. J Med Syst 1998;22(5):325-37.

4. Stead WW, Brame RG, Hammond WE, Jelovsek FR, Estes EH, Parker RT. A computerized obstetric medical record. Obstet Gynecol 1977;49(4):502-9.

5. Slack WV, Hicks GP, Reed CE, Van Cura LJ. A computer based medical-history system. N Engl J Med 1966;274(4):194-8.

6. Johnson KB, Cowan J. Clictate: a computer-based documentation tool for guideline-based care. J Med Syst 2002;26(1):47-60.

7. Kahn CE, Jr. Self-documenting structured reports using open information standards. Medinfo 1998;9(Pt 1):403-7.

8. McDonald CJ. The barriers to electronic medical record systems and how to overcome them. J Am Med Inform Assoc 1997;4(3):213-21.

9. Lum F, Schein O, Schachat AP, Abbott RL, Hoskins HD, Jr., Steinberg EP. Initial two years of experience with the AAO National Eyecare Outcomes Network (NEON) cataract surgery database. Ophthalmology 2000;107(4):691-7.

10. Poon AD, Fagan LM, Shortliffe EH. The PEN-Ivory project: exploring user-interface design for the selection of items from large controlled vocabularies of medicine.J AmMed Inform Assoc 1996;3(2):168-83. 
11. Rosenbloom ST, Kiepek W, Belletti J, Adams P, Shuxteau K, Johnson KB, Elkin PL, Shultz EK. Generating Complex Clinical Documents using Structured Entry and Reporting. Medinfo 2004.

12. Goldberg H, Morales A, McMillan D, Quinlan M. An Ontology-Driven Application to Improve the Prescription of Educational Resources to Parents of Premature Infants. EON 2003

13. Goldberg H, Morales A. Improving information prescription to parents of premature infants through an OWL-based knowledge mediator. Medinfo 2004;11(Pt 1):361-5.

14. Presidential Initiative on Consolidated Health Informatics, http://www.whitehouse.gov/ omb/egov/c-3-6-chi.html

15. Health Level 7, http://www.hl7.org

16. Snomed International, http://www.snomed.org

17. LOINC, http://www.regenstrief.org/loinc

18. International Classification of Diseases, Nine Revision, Clinical Modification - ICD-9CM, http://www.cdc.gov/nchs/about/otheract/icd9/abticd9.htm

19. RxNORM, http://www.nlm.nih.gov/research/umls/rxnorm_main.html

20. HUGO Gene Nomenclature Committee, http://www.gene.ucl.ac.uk/nomenclature

21. EPA Substance Registry System, http://www.epa.gov/srs 